\title{
Bioinformatics analysis of RNA sequencing data reveals multiple key genes in uterine corpus endometrial carcinoma
}

\author{
LIANG SHEN $^{1}$, MING LIU $^{1}$, WEI LIU $^{1}$, JING CUI $^{2}$ and CHANGZHONG LI ${ }^{1}$ \\ ${ }^{1}$ Department of Gynecology, Shandong Provincial Hospital Affiliated to Shandong University; \\ ${ }^{2}$ Department of Oral Surgery, Jinan Stomatology Hospital, Jinan, Shandong 250021, P.R. China
}

Received April 15, 2017; Accepted September 28, 2017

DOI: $10.3892 / \mathrm{ol} .2017 .7346$

\begin{abstract}
In the present study, the RNA sequencing (RNA-seq) data of uterine corpus endometrial carcinoma (UCEC) samples were collected and analyzed using bioinformatics tools to identify potential genes associated with the development of UCEC. UCEC RNA-seq data were downloaded from The Cancer Genome Atlas database. Differential analysis was performed using edgeR software. A false discovery rate $<0.01$ and $\log _{2}$ (fold change) $\mid>1$ were set as the cut-off criteria to screen for differentially expressed genes (DEGs). Differential gene co-expression analysis was performed using R/EBcoexpress package in $\mathrm{R}$. DEGs in the gene co-expression network were subjected to Gene Ontology analysis using the Database for Annotation, Visualization and Integration Discovery. Kyoto Encyclopedia of Genes and Genomes pathway enrichment analysis was also performed on the DEGs using KOBAS 2.0 software. The ConnectivityMap database was used to identify novel drug candidates. A total of 3,742 DEGs were identified among the 552 UCEC samples and 35 normal controls, and comprised 2,580 upregulated and 1,162 downregulated genes. A gene co-expression network consisting of 129 DEGs and 368 edges was constructed. Genes were associated with the cell cycle and the tumor protein p53 signaling pathway. Three modules were identified, in which genes were associated with the mitotic cell cycle, nuclear division and the $\mathrm{M}$ phase of the mitotic cell cycle. Multiple key hub genes were identified, including cell division cycle 20 , cyclin B2, non-SMC condensin I complex subunit H, BUB1 mitotic checkpoint serine/threonine kinase, cell division cycle associated 8, maternal embryonic leucine zipper kinase, MYB
\end{abstract}

Correspondence to: Dr Changzhong $\mathrm{Li}$, Department of Gynecology, Shandong Provincial Hospital Affiliated to Shandong University, 324 Jingwuweiqi Street, Jinan, Shandong 250021, P.R. China

E-mail: shenlang007@163.com

Key words: uterine corpus endometrial carcinoma, differentially expressed genes, gene co-expression network, small molecule drugs proto-oncogene like 2, TPX2, microtubule nucleation factor and non-SMC condensin I complex subunit G. In addition, the small molecule drug esculetin was implicated in the suppression of UCEC progression. Overall, the present study identified multiple key genes in UCEC and clinically relevant small molecule agents, thereby improving our understanding of UCEC and expanding perspectives on targeted therapy for this type of cancer.

\section{Introduction}

Endometrial cancer is the most commonly diagnosed female genital cancer and, in 2012, was ranked the fourth most common cancer in women worldwide, after breast, lung and colorectal cancer (1). In China, an estimated 63,400 new cases and 21,800 mortalities were reported for endometrial cancer in 2015 (2). Uterine corpus endometrial carcinoma (UCEC) is a common type of endometrial cancer. The incidence of UCEC increases with age and it is most frequently diagnosed in women aged between 45 and 65 years (3). Previous studies have sought to identify tumor biomarkers and have identified multiple UCEC biomarkers, including activated leukocyte cell adhesion molecule (4), sperm-associated antigen 9 (5), L1 cell adhesion molecule (6), progestogen-associated endometrial protein (7), heat shock protein family A (Hsp70) member 5 (8) and CD151 molecule (Raph blood group) (9). To enhance our understanding of UCEC and explore more effective and targeted therapies, the present study aimed to identify gene co-expression networks, hub genes and small molecule drugs associated with the development of UCEC.

The Cancer Genome Atlas (TCGA) is a comprehensive genomic database that holds data for $>20$ types of cancer obtained from thousands of patients. Data in TCGA includes whole-genome measurements of multiple genomic features, including DNA copy numbers, DNA methylation, and gene and microRNA expression, thereby assisting researchers in assessing cancer mechanisms at multiple molecular and regulatory levels (10). Furthermore, TGGA data is open access and available to all researchers in individual work settings.

In the present study, UCEC RNA sequencing data were collected and analyzed using bioinformatics tools. 
Differentially expressed genes (DEGs) were identified and a gene co-expression network was constructed, enabling the hub genes to be identified. Relevant small molecule drugs were also assessed. The results of the present study may provide novel insights into the pathogenesis of UCEC and thereby expand perspectives on the treatment of this type of tumor.

\section{Materials and methods}

Gene expression data. The RNA expression data (level 3) of UCEC patients and normal controls without UCEC were downloaded from the TCGA data portal up until December 2016 (http://cancergenome.nih.gov/) (11). A total of 552 UCEC samples and 35 normal control samples were included in the dataset. The exclusion criteria were as follows: A histological diagnosis not of UCEC; and samples without complete data for analysis.

DEG screening. The DEGs between UCEC and normal control tissues were screened using edgeR software (v..5; http://www. bioconductor.org/packages/release/bioc/html/edgeR.html). A false discovery rate $<0.01$ and $\log _{2}$ (fold change) $>1$ were set as the cut-off values to identify the DEGs.

Cluster analysis. Bidirectional hierarchical clustering according to the expression of the DEGs was performed using the pheatmap package(v.1.0.8;https://CRAN.R-project.org/package=pheatmap) in $\mathrm{R}$, as previously described (12). A heat map was used to represent the data (Fig. 1).

Gene co-expression network construction. Correlations among the DEGs were calculated using the R/EBcoexpress package in $\mathrm{R}$ (version 3.5; http://www.bioconductor. org/packages/release/bioc/html/EBcoexpress.html), as previously described (13). Genes with a correlation coefficient $>0.9$ were included in the gene co-expression network, which was visualized using Cytoscape software (version 3.5.1; http://www.cytoscape.org/).

Functional enrichment analysis. A Gene Ontology (GO) analysis (14) was performed on the DEGs in the gene co-expression network using the Database for Annotation, Visualization and Integration Discovery (http://david.abcc. ncifcrf.gov/) (15). $\mathrm{P}<0.05$ was set as the cut-off criteria. Kyoto Encyclopedia of Genes and Genomes (KEGG) pathway enrichment analysis (16) was performed using KOBAS 2.0 software (http://kobas.cbi.pku.edu.cn/) (17), with $\mathrm{P}<0.05$ set as the threshold.

Module analysis. Modules were identified using the MCODE plug-in in Cytoscape with the cut-off criteria of degree $\geq 2$ and k-core $\geq 3$. Each module was functionally annotated using the Cytoscape plug-in BiNGO based on hypergeometric distribution (adjusted P-value $<0.01)$.

Screening of relevant small molecule drugs. ConnectivityMap (cMap) (18) is a public database containing $>7,000$ expression profiles representing 1,309 compounds (www.broad.mit.edu/cmap/). Relevant small molecule drugs were predicted using the cMap tool and those with a Iscorel $>0.6$ were included.

\section{Results}

DEGs among UCEC and control samples. The differential expression analysis identified 3,742 DEGs, comprising 2,580 upregulated and 1,162 downregulated genes. The results of bidirectional hierarchical clustering of the 3,742 DEGs in the 587 samples (552 UCEC and 35 normal control samples) that were screened were provided (Fig. 1). The UCEC and control samples differed in gene expression pattern, suggesting that DEGs could distinguish between the two types of sample.

GO annotations of DEGs. The GO annotations of the DEGs were provided (Fig. 2). Cancer-associated processes, including cell adhesion, ion transport and biological adhesion, were among the significantly associated terms.

Gene co-expression network. Correlated genes with a correlation coefficient $>0.9$ were included in the gene co-expression network (Fig. 3). A total of 129 DEGs (nodes) and 368 edges (lines between nodes) were included, divided into 110 upregulated and 19 downregulated genes. The sizes of the nodes were proportional to the number of genes in the gene set, and a number of hub genes (nodes with degrees $\geq 8$ ) were highlighted, including cell division cycle 20 (CDC20), cyclin B2, non-SMC condensin I complex subunit $\mathrm{H}, \mathrm{BUB} 1$ mitotic checkpoint serine/threonine kinase, cell division cycle-associated 8 , maternal embryonic leucine zipper kinase, MYB proto-oncogene like 2, TPX2, microtubule nucleation factor and non-SMC condensin I complex subunit $\mathrm{G}$.

Co-expression network functional enrichment analysis. GO enrichment analysis identified 20 significantly overrepresented terms for gene function in the co-expression network (Table I), including $\mathrm{M}$ phase, nuclear division, mitosis and the $\mathrm{M}$ phase of the mitotic cell cycle. KEGG pathway enrichment analysis indicated that pathways such as those of the cell cycle, oocyte meiosis, progesterone-mediated oocyte maturation and tumor protein p53 signaling were significantly enriched among genes in the co-expression network (Table II).

Modules and functions. Three modules (designated A-C) were identified from the gene co-expression network (Fig. 4). Module A included 11 DEGs implicated in the mitotic cell cycle and cell cycle phases. Module B contained 6 DEGs associated with nuclear division. Module $\mathrm{C}$ comprised 7 DEGs associated with the $\mathrm{M}$ phase of the mitotic cell cycle (Table III).

Relevant small molecule drugs. A total of 7 small molecule drugs were identified, including esculetin, antazoline and isometheptene. Esculetin had the highest negative connectivity score compared with the 6 other small molecule drugs (-0.844; Table IV). 


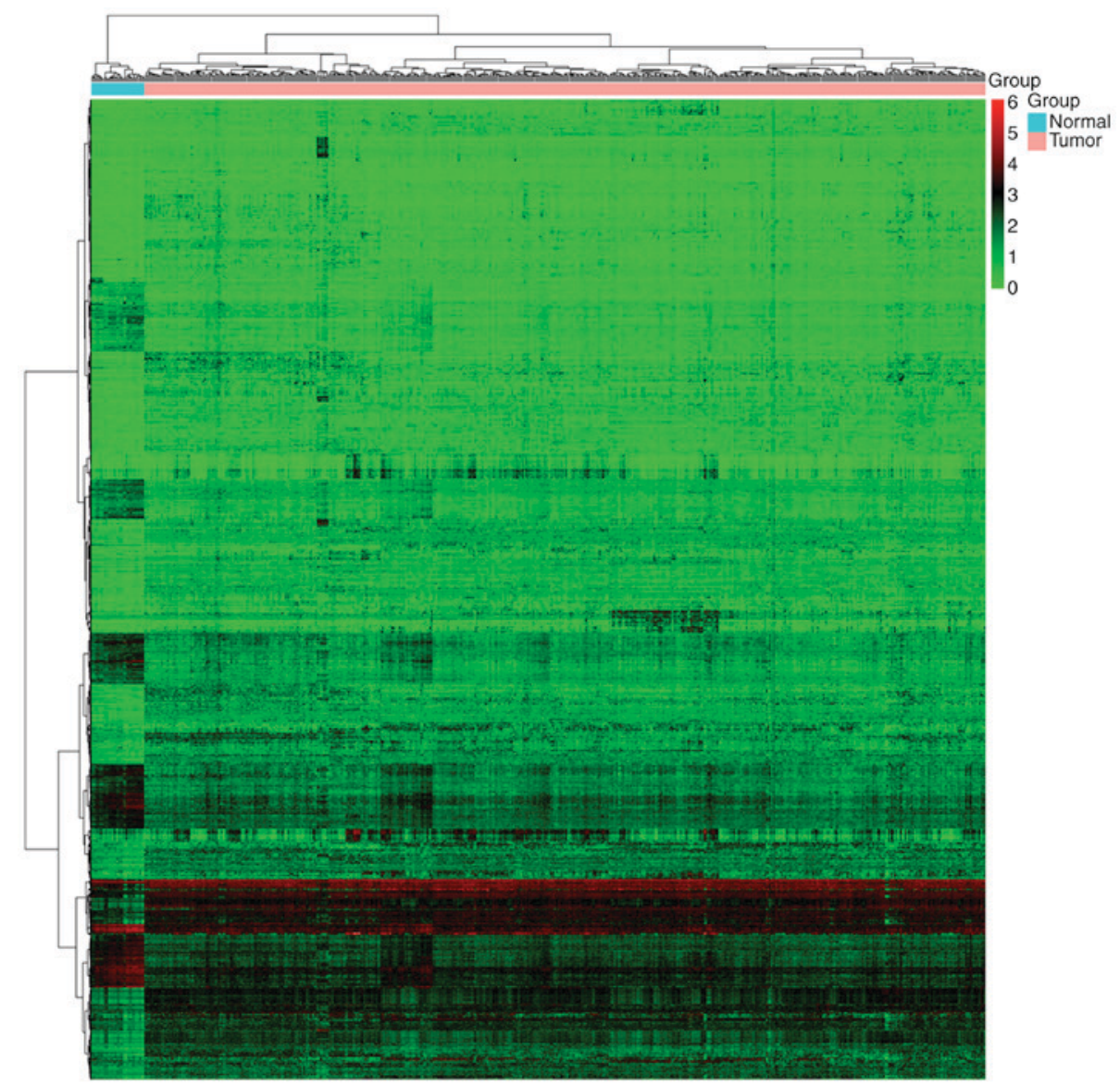

Figure 1. Bidirectional hierarchical clustering of the 3,742 differentially expressed genes within the 587 samples (552 uterine corpus endometrial carcinoma and 35 control) in the dataset.

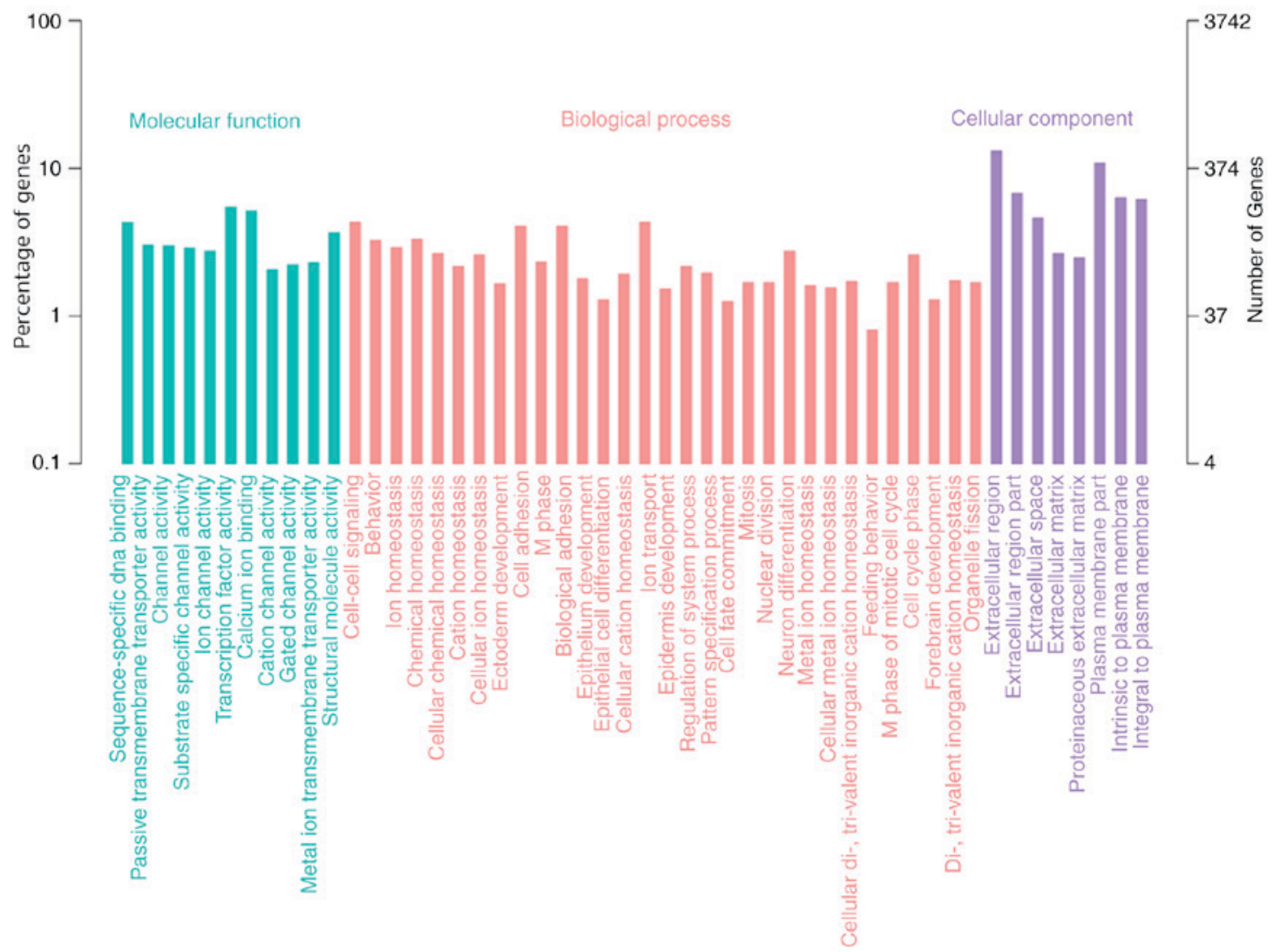

Figure 2. Gene Ontology annotations of the differentially expressed genes. 


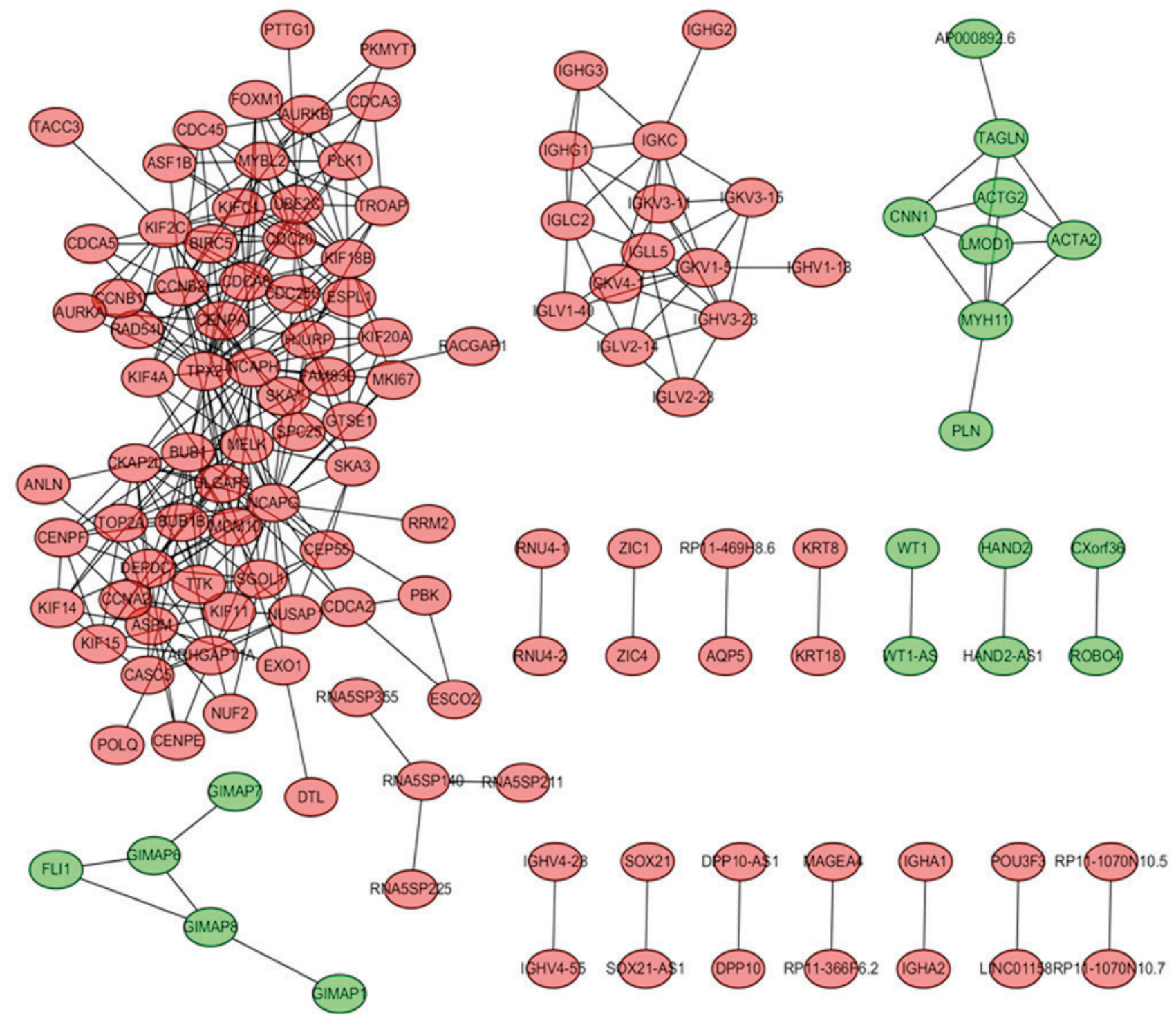

Figure 3. Gene co-expression network of differentially expressed genes. Red, upregulated genes; green, downregulated genes.

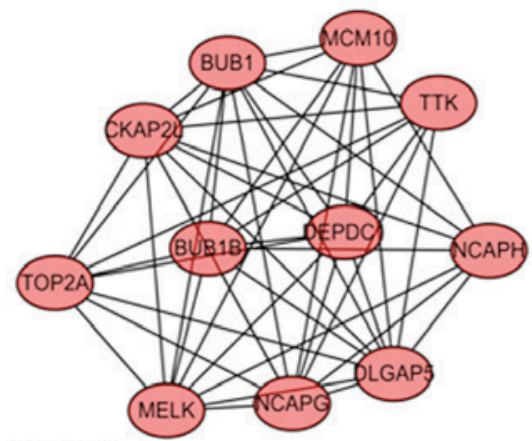

Module A

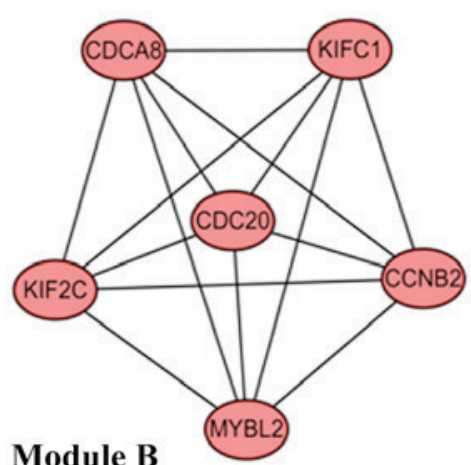

Module B

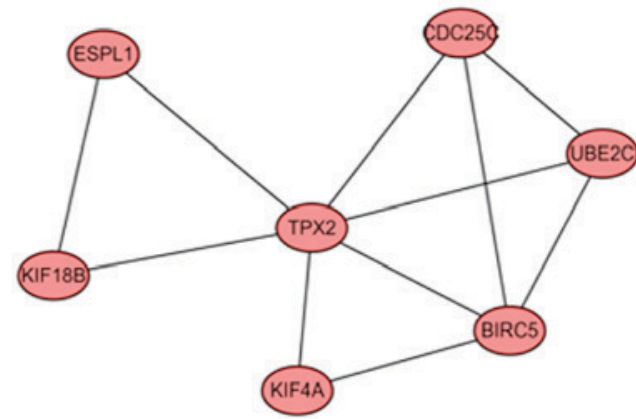

Module C

Figure 4. Modules identified from the gene co-expression network.

\section{Discussion}

The results of the present study indicated that multiple DEGs are associated with UCEC pathophysiology. Matrix metalloproteinases (MMPs) serve an important function in degrading the extracellular matrix and basement membrane components (19). Karahan et al identified that MMP-9 was expressed in a high percentage of primary 
Table I. GO terms significantly overrepresented in the genes from the gene co-expression network.

\begin{tabular}{|c|c|c|c|c|}
\hline GO category & GO term & Count & P-value & FDR \\
\hline GO:0000279 & M phase & 45 & $1.27 \times 10^{-46}$ & $1.93 \times 10^{-43}$ \\
\hline GO:0000280 & Nuclear division & 40 & $5.98 \times 10^{-46}$ & $9.07 \times 10^{-43}$ \\
\hline GO:0007067 & Mitosis & 40 & $5.98 \times 10^{-46}$ & $9.07 \times 10^{-43}$ \\
\hline GO:0000087 & M phase of the mitotic cell cycle & 40 & $1.27 \times 10^{-45}$ & $1.93 \times 10^{-42}$ \\
\hline GO:0048285 & Organelle fission & 40 & $3.20 \times 10^{-45}$ & $4.85 \times 10^{-42}$ \\
\hline GO:0022403 & Cell cycle phase & 46 & $1.33 \times 10^{-43}$ & $2.02 \times 10^{-40}$ \\
\hline GO:0000278 & Mitotic cell cycle & 43 & $3.22 \times 10^{-41}$ & $4.89 \times 10^{-38}$ \\
\hline GO:0007049 & Cell cycle & 53 & $2.40 \times 10^{-40}$ & $3.63 \times 10^{-37}$ \\
\hline GO:0022402 & Cell cycle process & 48 & $3.05 \times 10^{-40}$ & $4.62 \times 10^{-37}$ \\
\hline GO:0051301 & Cell division & 35 & $4.17 \times 10^{-33}$ & $6.33 \times 10^{-30}$ \\
\hline GO:0005819 & Spindle & 24 & $8.32 \times 10^{-27}$ & $9.68 \times 10^{-24}$ \\
\hline GO:0015630 & Microtubule cytoskeleton & 35 & $2.86 \times 10^{-26}$ & $3.33 \times 10^{-23}$ \\
\hline GO:0007059 & Chromosome segregation & 18 & $3.26 \times 10^{-21}$ & $4.94 \times 10^{-18}$ \\
\hline GO:0044430 & Cytoskeletal part & 37 & $1.25 \times 10^{-20}$ & $1.45 \times 10^{-17}$ \\
\hline GO:0005856 & Cytoskeleton & 41 & $5.11 \times 10^{-19}$ & $5.94 \times 10^{-16}$ \\
\hline GO:0043228 & Non-membrane-bounded organelle & 52 & $5.37 \times 10^{-18}$ & $6.24 \times 10^{-15}$ \\
\hline GO:0043232 & Intracellular non-membrane-bounded organelle & 52 & $5.37 \times 10^{-18}$ & $6.24 \times 10^{-15}$ \\
\hline GO:0000775 & Chromosome, centromeric region & 17 & $2.29 \times 10^{-17}$ & $2.67 \times 10^{-14}$ \\
\hline GO:0000793 & Condensed chromosome & 17 & $4.39 \times 10^{-17}$ & $5.10 \times 10^{-14}$ \\
\hline GO:0000779 & Condensed chromosome, centromeric region & 14 & $8.86 \times 10^{-17}$ & $1.33 \times 10^{-13}$ \\
\hline
\end{tabular}

GO, Gene Ontology; FDR, false discovery rate.

Table II. Kyoto Encyclopedia of Genes and Genomes pathways significantly overrepresented in the genes from the gene co-expression network.

\begin{tabular}{lcc}
\hline Term & Count & P-value \\
\hline hsa04110: Cell cycle & 13 & $3.65 \times 10^{-16}$ \\
hsa04114: Oocyte meiosis & 11 & $3.06 \times 10^{-13}$ \\
hsa04914: Progesterone-mediated & 7 & $3.60 \times 10^{-8}$ \\
oocyte maturation & & \\
hsa04115: p53 signaling pathway & 4 & $8.02 \times 10^{-5}$ \\
hsa05166: HTLV-I infection & 5 & $1.41 \times 10^{-3}$ \\
hsa04270: Vascular smooth muscle & 3 & $7.17 \times 10^{-3}$ \\
contraction & & \\
hsa04068: FoxO signaling pathway & 3 & $9.19 \times 10^{-3}$ \\
\hline
\end{tabular}

HTLV-I, human T-cell lymphotropic virus type 1; FoxO, forkhead box $\mathrm{O} ; \mathrm{p} 53$, tumor protein 53 .

endometrial carcinoma tissues and that MMP-9 expression was potentially associated with parameters of tumor aggressiveness (20). Furthermore, MMP-9 has been revealed to be overexpressed in UCEC and associated with UCEC progression (21). Topoisomerase $2 \alpha$, a key enzyme in DNA replication, has been implicated as a predictive marker in treating endometrial cancer with taxane-containing adjuvant chemotherapy (22). Enhancer of zeste homolog 2
(EZH2), a critical component of the polycomb repressive complex 2, is associated with cell proliferation, invasion, adhesion and metastasis in several cancer types (23), and is overexpressed in numerous types of cancer, including breast cancer (24), colorectal cancer (25), ovarian cancer (26) and nasopharyngeal cancer (27). A previous study indicated that EZH2 may predict a more aggressive endometrial carcinoma and may therefore potentially represent a therapeutic target for this type of cancer (28). Baculoviral inhibitor of apoptosis (IAP) repeat containing 5 gene (BIRC5), a member of the IAP gene family, may inhibit the activation of caspase to negatively regulate apoptosis (29). In endometrial cancer, increased BIRC5 expression has been associated with poor progression-free survival and may serve as an independent prognostic factor (30).

It is well established that CDC20 is an essential developmental gene; disrupting CDC20 function in mice induces mortality in embryos (31). Previous studies have also suggested that CDC20 functions oncogenically in human tumorigenesis. CDC20 serves important functions in the cell cycle (32), cell apoptosis (33) and in targeting downstream substrates for ubiquitination and subsequent degradation (34). Furthermore, $\mathrm{CDC} 20$ has been revealed to be upregulated and may represent a useful prognostic biomarker in endometrial cancer (35).

In the present study, esculetin was identified as a significant small molecule drug in the development of UCEC. Esculetin (6,7-dihydroxycoumarin), a coumarin derived from natural plants, inhibits cancer cells from proliferating and induces multiple types of human cancer cells to 
Table III. Functional terms of the three modules.

\begin{tabular}{|c|c|c|c|}
\hline GO-ID & P-value & FDR & No. of genes \\
\hline \multicolumn{4}{|l|}{ Module A } \\
\hline GO:0000279 M phase & $9.60 \times 10^{-7}$ & $1.12 \times 10^{-3}$ & 6 \\
\hline GO:0000278 mitotic cell cycle & $1.71 \times 10^{-6}$ & $2.01 \times 10^{-3}$ & 6 \\
\hline GO:0022403 cell cycle phase & $2.98 \times 10^{-6}$ & $3.50 \times 10^{-3}$ & 6 \\
\hline GO:0007067 mitosis & $8.04 \times 10^{-6}$ & $9.43 \times 10^{-3}$ & 5 \\
\hline GO:0000280 nuclear division & $8.04 \times 10^{-6}$ & $9.43 \times 10^{-3}$ & 5 \\
\hline GO:0000087 M phase of mitotic cell cycle & $8.64 \times 10^{-6}$ & $1.01 \times 10^{-2}$ & 5 \\
\hline GO:0048285 organelle fission & $9.43 \times 10^{-6}$ & $1.11 \times 10^{-2}$ & 5 \\
\hline GO:0022402 cell cycle process & $1.37 \times 10^{-5}$ & $1.60 \times 10^{-2}$ & 6 \\
\hline GO:0007059 chromosome segregation & $1.69 \times 10^{-5}$ & $1.98 \times 10^{-2}$ & 4 \\
\hline GO:0000793 condensed chromosome & $3.41 \times 10^{-5}$ & $3.10 \times 10^{-2}$ & 4 \\
\hline GO:0005819 spindle & $5.04 \times 10^{-5}$ & $4.59 \times 10^{-2}$ & 4 \\
\hline GO:0005694 chromosome & $5.32 \times 10^{-5}$ & $4.83 \times 10^{-2}$ & 5 \\
\hline \multicolumn{4}{|l|}{ Module B } \\
\hline GO:0000280 nuclear division & $3.36 \times 10^{-7}$ & $3.63 \times 10^{-4}$ & 5 \\
\hline GO:0007067 mitosis & $3.36 \times 10^{-7}$ & $3.63 \times 10^{-4}$ & 5 \\
\hline GO:0000087 M phase of mitotic cell cycle & $3.61 \times 10^{-7}$ & $3.90 \times 10^{-4}$ & 5 \\
\hline GO:0048285 organelle fission & $3.95 \times 10^{-7}$ & $4.27 \times 10^{-4}$ & 5 \\
\hline GO:0000279 M phase & $1.69 \times 10^{-6}$ & $1.82 \times 10^{-3}$ & 5 \\
\hline GO:0000278 mitotic cell cycle & $2.69 \times 10^{-6}$ & $2.93 \times 10^{-3}$ & 5 \\
\hline GO:0015630 microtubule cytoskeleton & $3.37 \times 10^{-6}$ & $2.94 \times 10^{-3}$ & 5 \\
\hline GO:0022403 cell cycle phase & $4.22 \times 10^{-6}$ & $4.56 \times 10^{-3}$ & 5 \\
\hline GO:0022402 cell cycle process & $1.46 \times 10^{-5}$ & $1.57 \times 10^{-2}$ & 5 \\
\hline \multicolumn{4}{|l|}{ Module C } \\
\hline GO:0000280 nuclear division & $3.36 \times 10^{-7}$ & $3.63 \times 10^{-4}$ & 5 \\
\hline GO:0007067 mitosis & $3.36 \times 10^{-7}$ & $3.63 \times 10^{-4}$ & 5 \\
\hline GO:0000087 M phase of mitotic cell cycle & $3.61 \times 10^{-7}$ & $3.90 \times 10^{-4}$ & 5 \\
\hline GO:0048285 organelle fission & $3.95 \times 10^{-7}$ & $4.27 \times 10^{-4}$ & 5 \\
\hline GO:0000279 M phase & $1.69 \times 10^{-6}$ & $1.82 \times 10^{-3}$ & 5 \\
\hline GO:0000278 mitotic cell cycle & $2.69 \times 10^{-6}$ & $2.91 \times 10^{-3}$ & 5 \\
\hline GO:0015630 microtubule cytoskeleton & $3.37 \times 10^{-6}$ & $2.94 \times 10^{-3}$ & 5 \\
\hline GO:0022403 cell cycle phase & $4.22 \times 10^{-6}$ & $4.56 \times 10^{-3}$ & 5 \\
\hline GO:0022402 cell cycle process & $1.46 \times 10^{-5}$ & $1.57 \times 10^{-2}$ & 5 \\
\hline
\end{tabular}

GO-ID, Gene Ontology identification; FDR, false discovery rate.

Table IV. Relevant small molecule drugs.

\begin{tabular}{lcc}
\hline cMap name & Correlation & P-value \\
\hline Esculetin & -0.844 & $7.55 \times 10^{-3}$ \\
Antazoline & -0.729 & $1.07 \times 10^{-2}$ \\
Isometheptene & -0.722 & $1.20 \times 10^{-2}$ \\
Oxamniquine & 0.717 & $1.31 \times 10^{-2}$ \\
Pyrimethamine & -0.642 & $1.49 \times 10^{-2}$ \\
Carmustine & -0.795 & $1.75 \times 10^{-2}$ \\
Cefapirin & 0.636 & $4.28 \times 10^{-2}$ \\
\hline
\end{tabular}

cMap, connectivity map. apoptose, including breast $(36)$, colon $(37,38)$ and gastric cancer (39), malignant melanoma (40), hepatocellular carcinoma $(41,42)$ and oral squamous cancer $(43,44)$. Esculetin has also been reported to assist in inducing cancer cells to apoptose $(45,46)$. In the present study, esculetin had a higher negative connectivity score compared with the 6 other small molecule drugs, suggesting that esculetin could inhibit UCEC from progressing. To the best of our knowledge, no study has yet reported on the association between esculetin and endometrial cancer. Verifying whether esculetin represents a novel therapeutic agent in treating UCEC requires further studies. Overall, the present study identified multiple key genes in UCEC and clinically relevant small molecule agents, thereby improving our understanding of UCEC 
and expanding perspectives on targeted therapy for this type of cancer.

\section{Acknowledgements}

The present study was supported by the National Natural Science Foundation of China (grant no. 81671434).

\section{References}

1. Torre LA, Bray F, Siegel RL, Ferlay J, Lortet-Tieulent J and Jemal A Global cancer statistics, 2012. CA Cancer J Clin 65: 87-108, 2015.

2. Chen W, Zheng R, Baade PD, Zhang S, Zeng H, Bray F, Jemal A, Yu XQ and He J: Cancer statistics in China, 2015. CA Cancer J Clin 66: 115-132, 2016.

3. Rutgers JK: Update on pathology, staging and molecular pathology of endometrial (uterine corpus) adenocarcinoma. Future Oncol 11: 3207-3218, 2015.

4. Devis L, Moiola CP, Masia N, Martinez-Garcia E, Santacana M, Stirbat TV, Brochard-Wyart F, García Á, Alameda F, Cabrera S, et al: Activated leukocyte cell adhesion molecule (ALCAM) is a marker of recurrence and promotes cell migration, invasion, and metastasis in early-stage endometrioid endometrial cancer. J Pathol 241: 475-487, 2017.

5. Baser E, Togrul C, Ozgu E, Ayhan S, Caglar M, Erkaya S and Gungor T: Sperm-associated antigen 9 is a promising marker for early diagnosis of endometrial cancer. Asian Pac J Cancer Prev 14: 7635-7638, 2013

6. Smogeli E, Davidson B, Cvancarova M, Holth A, Katz B, Risberg B, Kristensen G and Lindemann K: L1CAM as a prognostic marker in stage I endometrial cancer: A validation study. BMC Cancer 16: 596, 2016.

7. Lenhard M, Heublein S, Kunert-Keil C, Vrekoussis T, Lomba I, Ditsch N, Mayr D, Friese K and Jeschke U: Immunosuppressive Glycodelin A is an independent marker for poor prognosis in endometrial cancer. BMC Cancer 13: 616, 2013.

8. Matsuo K, Gray MJ, Yang DY, Srivastava SA, Tripathi PB, Sonoda LA, Yoo EJ, Dubeau L, Lee AS and Lin YG: The endoplasmic reticulum stress marker, glucose-regulated protein-78 (GRP78) in visceral adipocytes predicts endometrial cancer progression and patient survival. Gynecol Oncol 128: 552-559, 2013.

9. Voss MA, Gordon N, Maloney S, Ganesan R, Ludeman L, McCarthy K, Gornall R, Schaller G, Wei W, Berditchevski F and Sundar S: Tetraspanin CD151 is a novel prognostic marker in poor outcome endometrial cancer. Br J Cancer 104: 1611-1618, 2011.

10. Xu Y, Zhu Y, Müller P, Mitra R and Ji Y: Characterizing cancer-specific networks by integrating TCGA data. Cancer Inform 13 (Suppl 2): S125-S131, 2014.

11. Cancer Genome Atlas Research Network: Comprehensive genomic characterization defines human glioblastoma genes and core pathways. Nature 455: 1061-1068, 2008.

12. Wang L, Cao C, Ma Q, Zeng Q, Wang H, Cheng Z, Zhu G, Qi J, $\mathrm{Ma} \mathrm{H}$, Nian $\mathrm{H}$ and Wang Y: RNA-seq analyses of multiple meristems of soybean: Novel and alternative transcripts, evolutionary and functional implications. BMC Plant Biol 14: 169, 2014.

13. Dawson JA, Ye S and Kendziorski C: R/EBcoexpress: An empirical Bayesian framework for discovering differential co-expression. Bioinformatics 28: 1939-1940, 2012.

14. Hulsegge I, Kommadath A and Smits MA: Globaltest and GOEAST: Two different approaches for Gene Ontology analysis. BMC Proc 3 (Suppl 4): S10, 2009.

15. Huang da W, Sherman BT and Lempicki RA: Systematic and integrative analysis of large gene lists using DAVID bioinformatics resources. Nat Protoc 4: 44-57, 2009.

16. Altermann E and Klaenhammer TR: PathwayVoyager: Pathway mapping using the Kyoto Encyclopedia of Genes and Genomes (KEGG) database. BMC Genomics 6: 60, 2005.

17. Wu J, Mao X, Cai T, Luo J and Wei L: KOBAS server: A web-based platform for automated annotation and pathway identification. Nucleic Acids Res 34 (Web Server Issue): W720-W724, 2006.

18. Lamb J, Crawford ED, Peck D, Modell JW, Blat IC, Wrobel MJ, Lerner J, Brunet JP, Subramanian A, Ross KN, et al: The Connectivity Map: Using gene-expression signatures to connect small molecules, genes, and disease. Science 313: 1929-1935, 2006.
19. Knapinska AM, Estrada CA and Fields GB: The roles of matrix metalloproteinases in pancreatic cancer. Prog Mol Biol Transl Sci 148: 339-354, 2017.

20. Karahan N, Güney M, Baspinar S, Oral B, Kapucuoglu N and Mungan T: Expression of gelatinase (MMP-2 and MMP-9) and cyclooxygenase-2 (COX-2) in endometrial carcinoma. Eur J Gynaecol Oncol 28: 184-188, 2007.

21. Yu F, Jiang Q, Zhou Y, Yang Z, Yu X, Wang H, Liu Z, Wang L, Fang $\mathrm{W}$ and Guo $\mathrm{S}$ : Abnormal expression of matrix metalloproteinase-9 (MMP9) correlates with clinical course in Chinese patients with endometrial cancer. Dis Markers 32: 321-327, 2012.

22. Ito F, Furukawa $\mathrm{N}$ and Nakai T: Evaluation of TOP2A as a predictive marker for endometrial cancer with taxane-containing adjuvant chemotherapy. Int J Gynecol Cancer 26: 325-330, 2016.

23. Shen L, Cui J, Liang S, Pang Y and Liu P: Update of research on the role of EZH2 in cancer progression. Onco Targets Ther 6: 321-324, 2013.

24. Yamaguchi H, Du Y, Nakai K, Ding M, Chang SS, Hsu JL, Yao J, Wei Y, Nie L, Jiao S, et al: EZH2 contributes to the response to PARP inhibitors through its PARP-mediated poly-ADP ribosylation in breast cancer. Oncogene 2017.

25. Lian Y, Yan C, Ding J, Xia R, Ma Z, Hui B, Ji H, Zhou J and Wang K: A novel lncRNA, LL22NC03-N64E9.1, represses KLF2 transcription through binding with EZH2 in colorectal cancer. Oncotarget 8: 59435-59445, 2017.

26. Zhou F, Chen J and Wang H: MicroRNA-298 inhibits malignant phenotypes of epithelial ovarian cancer by regulating the expression of EZH2. Oncol Lett 12: 3926-3932, 2016.

27. Yu L, Lu J, Zhang B, Liu X, Wang L, Li SY, Peng XH, Xu X, Tian WD and Li XP: miR-26a inhibits invasion and metastasis of nasopharyngeal cancer by targeting EZH2. Oncol Lett 5: 1223-1228, 2013 .

28. Jia N, Li Q, Tao X, Wang J, Hua K and Feng W: Enhancer of zeste homolog 2 is involved in the proliferation of endometrial carcinoma. Oncol Lett 8: 2049-2054, 2014.

29. Ma F, Zhang H, Zhai Y, Huang W, Zhao C, Ou S, Zhou H, Yuan W, Wang Z, Wang H, et al: Functional polymorphism $-31 \mathrm{C} / \mathrm{G}$ in the promoter of BIRC5 gene and risk of nasopharyngeal carcinoma among chinese. PLoS One 6: e16748, 2011.

30. Chuwa AH, Sone K, Oda K, Ikeda Y, Fukuda T, Wada-Hiraike O, Inaba K, Makii C, Takeuchi M, Oki S, et al: Significance of survivin as a prognostic factor and a therapeutic target in endometrial cancer. Gynecol Oncol 141: 564-569, 2016.

31. Li M, York JP and Zhang P: Loss of Cdc20 causes a securin-dependent metaphase arrest in two-cell mouse embryos. Mol Cell Biol 27: 3481-3488, 2007.

32. Hadjihannas MV, Bernkopf DB, Brückner M and Behrens J: Cell cycle control of $\mathrm{Wnt} / \mathrm{b}$-catenin signalling by conductin/axin2 through CDC20. EMBO Rep 13: 347-354, 2012.

33. Harley ME, Allan LA, Sanderson HS and Clarke PR: Phosphorylation of Mcl-1 by CDK1-cyclin B1 initiates its Cdc20-dependent destruction during mitotic arrest. EMBO J 29: 2407-2420, 2010.

34. Xie Q, Wu Q, Mack SC, Yang K, Kim L, Hubert CG, Flavahan WA, Chu C, Bao S and Rich JN: CDC20 maintains tumor initiating cells. Oncotarget 6: 13241-13254, 2015.

35. Gayyed MF, El-Maqsoud NM, Tawfiek ER, El Gelany SA and Rahman MF: A comprehensive analysis of CDC20 overexpression in common malignant tumors from multiple organs: Its correlation with tumor grade and stage. Tumour Biol 37: 749-762, 2016.

36. Chang HT, Chou CT, Lin YS, Shieh P, Kuo DH, Jan CR and Liang WZ: Esculetin, a natural coumarin compound, evokes $\mathrm{Ca}(2+)$ movement and activation of $\mathrm{Ca}(2+)$-associated mitochondrial apoptotic pathways that involved cell cycle arrest in ZR-75-1 human breast cancer cells. Tumour Biol 37: 4665-4678, 2016.

37. Kim AD, Han X, Piao MJ, Hewage SR, Hyun CL, Cho SJ and Hyun JW: Esculetin induces death of human colon cancer cells via the reactive oxygen species-mediated mitochondrial apoptosis pathway. Environ Toxicol Pharmacol 39: 982-989, 2015.

38. Kim AD, Madduma Hewage SR, Piao MJ, Kang KA, Cho SJ and Hyun JW: Esculetin induces apoptosis in human colon cancer cells by inducing endoplasmic reticulum stress. Cell Biochem Funct 33: 487-494, 2015.

39. Pan H, Wang BH, Lv W, Jiang Y and He L: Esculetin induces apoptosis in human gastric cancer cells through a cyclophilin D-mediated mitochondrial permeability transition pore associated with ROS. Chem Biol Interact 242: 51-60, 2015. 
40. Jeon YJ, Jang JY, Shim JH, Myung PK and Chae JI: Esculetin, a coumarin derivative, exhibits anti-proliferative and pro-apoptotic activity in G361 human malignant melanoma. J Cancer Prev 20 106-112, 2015.

41. Wang J, Lu ML, Dai HL, Zhang SP, Wang HX and Wei N: Esculetin, a coumarin derivative, exerts in vitro and in vivo antiproliferative activity against hepatocellular carcinoma by initiating a mitochondrial-dependent apoptosis pathway. Braz J Med Biol Res 48: 245-253, 2015.

42. Kuo HC, Lee HJ, Hu CC, Shun HI and Tseng TH: Enhancement of esculetin on Taxol-induced apoptosis in human hepatoma HepG2 cells. Toxicol Appl Pharmacol 210: 55-62, 2006.

43. Cho JH, Shin JC, Cho JJ, Choi YH, Shim JH and Chae JI: Esculetin (6,7-dihydroxycoumarin): A potential cancer chemopreventive agent through suppression of $\mathrm{Spl}$ in oral squamous cancer cells. Int J Oncol 46: 265-271, 2015.
44. Kok SH, Yeh CC, Chen ML and Kuo MY: Esculetin enhances TRAIL-induced apoptosis through DR5 upregulation in human oral cancer SAS cells. Oral Oncol 45: 1067-1072, 2009.

45. Arora R, Sawney S, Saini V, Steffi C, Tiwari M and Saluja D: Esculetin induces antiproliferative and apoptotic response in pancreatic cancer cells by directly binding to KEAP1. Mol Cancer 15: 64, 2016.

46. Jeon YJ, Cho JH, Lee SY, Choi YH, Park H, Jung S, Shim JH and Chae JI: Esculetin induces apoptosis through EGFR/PI3K/Akt signaling pathway and nucleophosmin relocalization. J Cell Biochem 117: 1210-1221, 2016. 